\title{
Nefrectomía radical laparoscópica
}

\author{
Rubio Briones J, Iborra Juan I, Casanova Ramón-Borja J, Solsona Narbón E. \\ Servicio de Urología. Instituto Valenciano de Oncología. Valencia.
}

Actas Urol Esp 2006; 30 (5): 479-491

\section{RESUMEN}

NEFRECTOMÍA RADICAL LAPAROSCÓPICA

Revisamos en este artículo los distintas abordajes quirúrgicos para realizar una nefrectomía radical laparoscópica, la vía transperitoneal, la retroperitoneal, así como la mano-asistida.

Describimos las ventajas e inconvenientes de cada una de estas alternativas así como resumimos los citas bibliográficas más importantes en la literatura médica con respecto a las mismas.

A pesar de que es una cirugía con una corta existencia, menos de 15 años, ha conseguido convertirse en un estándar de tratamiento y hoy puede considerarse en muchos centros como la cirugía de elección en tumores de riñón T1 y T2.

Palabras clave: Laparoscopia. Nefrectomía radical.

\section{ABSTRACT}

\section{RADICAL LAPAROSCOPIC NEPHRECTOMY}

In this article, we review the different surgical approaches to carry out radical laparoscopic nephrectomy: transperitoneal approach, retroperitoneal approach and hand-assisted approach.

We describe the advantages and drawbacks of each alternative and summarize the most important references in the medical literature.

In spite of this being a relatively new surgical approach, less than 15 years old, it has become a standard treatment and, today, is considered as the elective surgical treatment for T1 and T2 renal tumours in many centres.

Keywords: Laparoscopy. Radical nephrectomy.

\section{INTRODUCCIÓN HISTÓRICA}

La nefrectomía radical laparoscópica (NRL) tiene apenas 15 años de vida; en junio de 1990 fue el Dr. Clayman ${ }^{1}$ quien realizó por primera vez esta cirugía en EEUU. Su desarrollo fue paulatino pero implacable hasta nuestros días, de tal forma que en la última versión de las guidelines de la Asociación Europea de Urología publicadas en agosto de 2004 se le considera la técnica de elección para los carcinomas renales estadios $\mathrm{T} 1$ y T2, considerándola una técnica absolutamente factible, con un nivel de evidencia $2 b$ (basada en al menos un estudio o más bien diseñados y quasi experimentales) y con un nivel de reco- mendación C (hacerla pese a la ausencia de estudios especialmente diseñados y de buena calidad a tal fin).

Otros hechos reseñables en su historia fueron la descripción por Gaur de su balón para crear el espacio retroperitoneal en 1993 y por tanto el inicio del desarrollo de la NRL por vía retroperitoneal ${ }^{2}$ y la estandarización de la NRL mano-asistida en 1997 por Nakada ${ }^{3}$.

El año 1999 fue sin duda importante en la historia de la NRL; se publicaron 4 estudios que en total comparaban, aunque no de forma prospectiva ni randomizada, 161 casos de NRL frente a 121 de NR abierta. Pese a ser estudios absolu- 
tamente independientes, los 4 coincidieron en las mismas conclusiones; la NRL suponía un menor trauma quirúrgico que la abierta, lo que se traducía en una menor necesidad de analgésicos, una menor pérdida sanguínea y unas estancias y convalecencias más cortas, además de las inherentes ventajas cosméticas.

Inicialmente los detractores de la NRL argumentaron la ausencia de resultados oncológicos a largo plazo y el mayor tiempo y coste quirúrgicos. Posteriormente se expondrán los resultados oncológicos de las primeras series a 5 años, absolutamente comparables a la vía abierta. Respecto al tiempo, la experiencia lo equipara al de abierta. Por último, el coste es también directamente proporcional a la experiencia en función del tiempo y material utilizados y está sujeto a una serie de condicionamientos locales y del sistema sanitario que hacen realmente complicado su comparación objetiva, pero ya existen numerosos artículos que, incluso utilizando material desechable (por ley en EEUU), justifican un coste global más barato para la NRL.

Sin embargo, y pese al tirón de esta técnica, estamos asistiendo en estos momentos al momento álgido de su difusión a nivel mundial. En 2002, una encuesta realizada entre los urólogos de California objetivó que éstos realizaban en total 0,5 NRL frente a 4,5 NR abiertas por año. Realizando una búsqueda en el medline cruzando las palabras laparoscopic y nephrectomy en el año 2004, sólo 4 artículos de 396 (1\%) son originarios de nuestro país ${ }^{4-7}$. En instituciones con larga experiencia en cirugía laparoscópica, ya se considera que la NRL ha sustituido como técnica de elección a la nefrectomía radical abierta para tumores cT1 y cT2. En nuestro país somos muchos grupos los que ya realizamos la NRL como técnica de elección en tumores T1-T2 y patología benigna y muchos más los que están iniciando sus curvas de aprendizaje.

\section{TÉCNICA}

El debate entre la elección de la vía retroperitoneal o transperitoneal para la NRL se estableció desde los albores de esta técnica. Como casi todo en Medicina, la orientación que actualmente se le da al tema en distintos foros laparoscópicos es que la realización de la NRL "estándar" por una u otra vía es una cuestión de escuela, pero que ambas técnicas jamás deben ser competitivas y sí complementarias, pues pueden existir determinadas indicaciones específicas para cada una de ellas.

Recientemente se han publicado 2 estudios prospectivos al respecto sobre 40 y 102 pacientes en donde las dos vías se han realizado por grupos expertos al 50\% aproximadamente y de forma aleatoria ${ }^{8,9}$. En ambos estudios no se objetivan diferencias estadísticamente significativas en resultados oncológicos, pérdidas sanguíneas, complicaciones ni estancias o convalecencias postquirúrgicas. Se reconoce que en la vía retroperitoneal el control hiliar y el tiempo quirúrgico es ligeramente más rápido, recomendándola para los pacientes obesos y para aquellos con cirugías abdominales previas. La vía transperitoneal, por el contrario, se recomienda para aquellos pacientes que han sufrido cirugia retroperitoneal, para tumores grandes o NRL citorreductoras en pacientes metastásicos, para nefrectomías parciales, de donante vivo y cuando se vaya a utilizar robótica o dispositivos mano asistida. En los pacientes con tumores renales cT2 se aconseja llevarse un parche anterior de peritoneo por la posibilidad de márgenes positivos a dicho nivel ${ }^{10}$.

\section{Via transperitoneal}

Como la cirugía abierta, la técnica de la NRL no es igual en manos de ningún cirujano; cada uno tiene sus preferencias y trucos. A continuación se exponen los distintos pasos que nuestro grupo realiza para la misma, pero a los mismos podrían añadirse muchas variantes completamente válidas.

a) Colocación del paciente y cirujanos (Fig. 1) lumbotomía clásica. Se desaconseja colocar un rodillo insuflado para partir al paciente porque puede rechazar el paquete intestinal hacia el flanco dificultando la visibilidad de la cámara. El cirujano se coloca de pie frente al abdomen del paciente, su asistente es preferible que esté sentado y la instrumentista debe tener accesibilidad a manejar el $4^{\circ}$ trócar. Vigilar la fijación y almohadillado del paciente.

b) Creación del pneumoperitoneo y colocación de trócares; normalmente se usan 4 trócares. $\mathrm{El}$ 


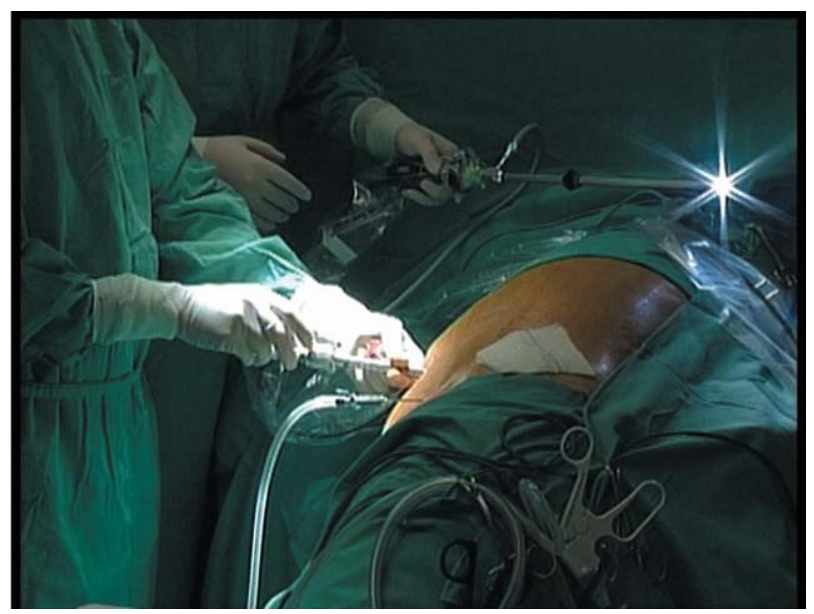

FIGURA 1

primero de $10 \mathrm{~mm}$ se sitúa en condiciones estándar paraumbilical en la línea externa del recto del abdomen y por él se colocará la óptica. Esta puede ser de $0^{\circ}$ o $30^{\circ}$, aunque la disponibilidad de ésta última facilita toda la cirugía retroperitoneal. En la fosa ilíaca y en hipocondrio ipsilaterales se colocan el $2^{\circ}$ y $3^{\text {er }}$ trócares, asignando a la mano dominante del cirujano el trócar de $12 \mathrm{~mm}$ que permita el uso de endo-GIAs y a la otra uno de $5 \mathrm{~mm}$. El $4^{\circ}$ trócar, de $5 \mathrm{~mm}$, se coloca en la línea axilar anterior y lo usa el ayudante/instrumentista.

c) Decolación colon derecho/izquierdo (Fig. 2).

d) Creación plano entre cara anterior renal y colon; (Fig. 3) Maniobra de Köcher en el lado derecho. Desplazamiento del paquete espleno-pancreático en el izquierdo (Fig. 4).

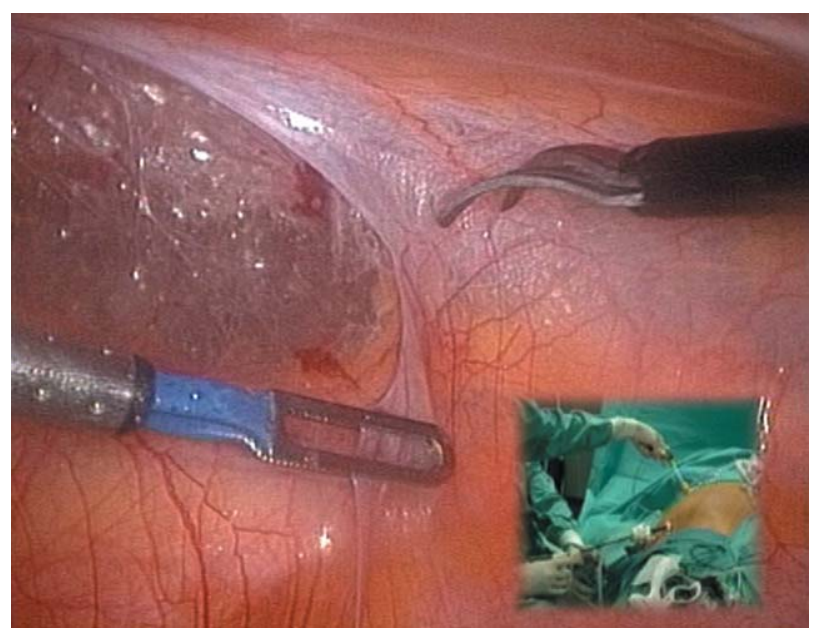

FIGURA 2

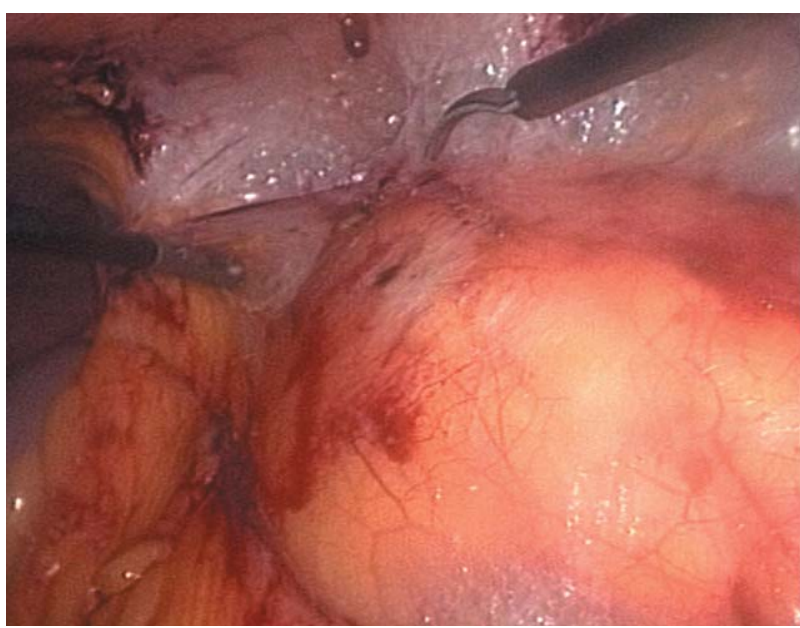

FIGURA 3

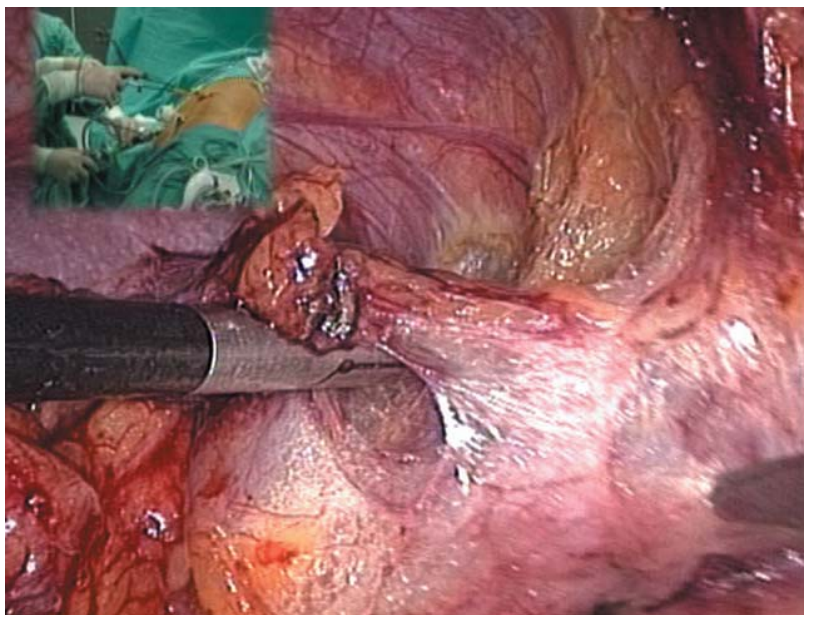

FIGURA 4

e) Individualización de vena gonadal y uréter; (Fig. 5). A excepción de variantes, la gonadal siempre se encontrará primero y en una situación más externa a la línea media en lumbotomía. Es una pista clave para localizar la vena renal izquierda o la cava.

f) Acceso ascendente hacia el hilio renal; mediante esta maniobra se pueden encontrar pedículos polares inferiores que deben ser correctamente controlados (Fig. 6). En laparoscopia es frecuente visualizar la arteria gonadal, que se puede controlar con bipolar o clips.

g) Identificación, control y sección de arteria $y$ vena; (Figs. 7, 8 y 9). La disección de los elementos del hilio debe hacerse siguiendo los principios de cirugía abierta. En nuestro grupo solemos clipar la arteria con 3 hem-o-locks, dejando 2 proxi- 


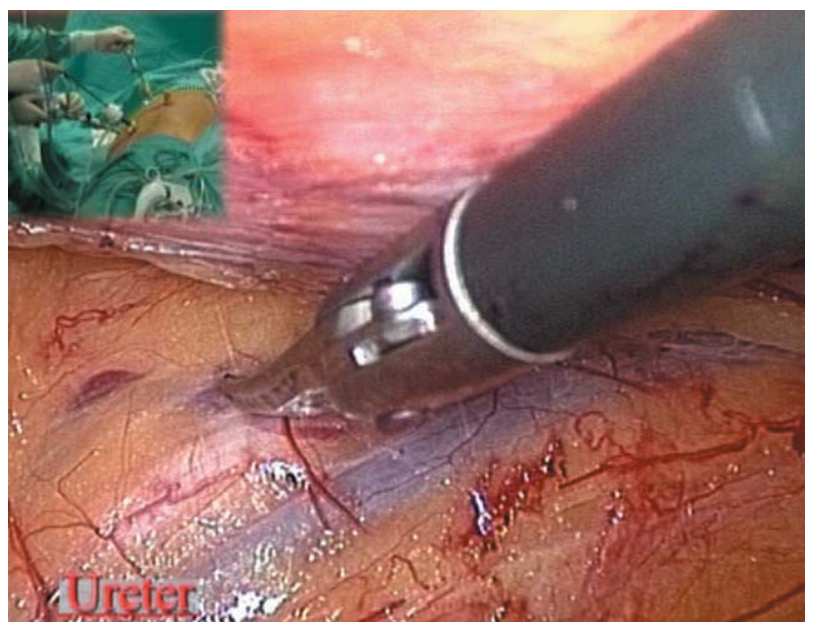

\section{FIGURA 5}

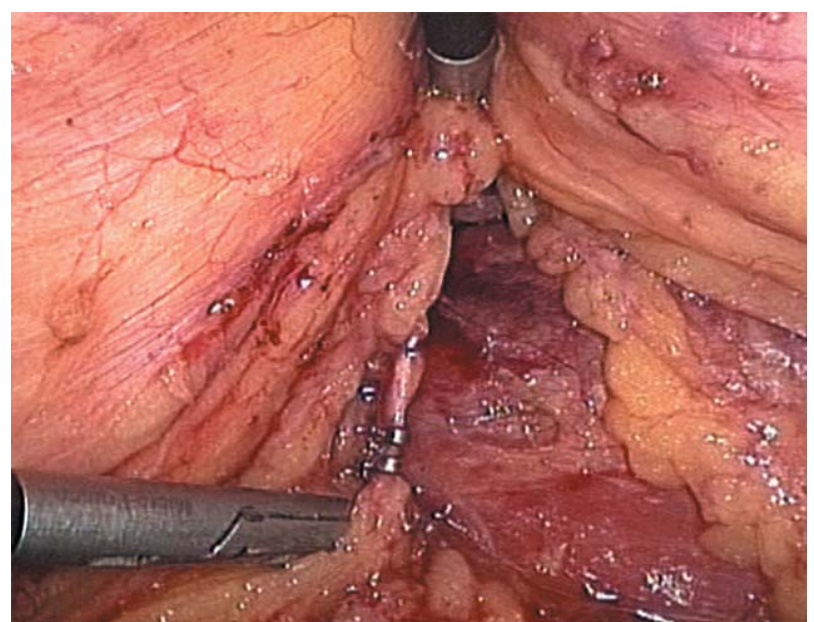

\section{FIGURA 6}

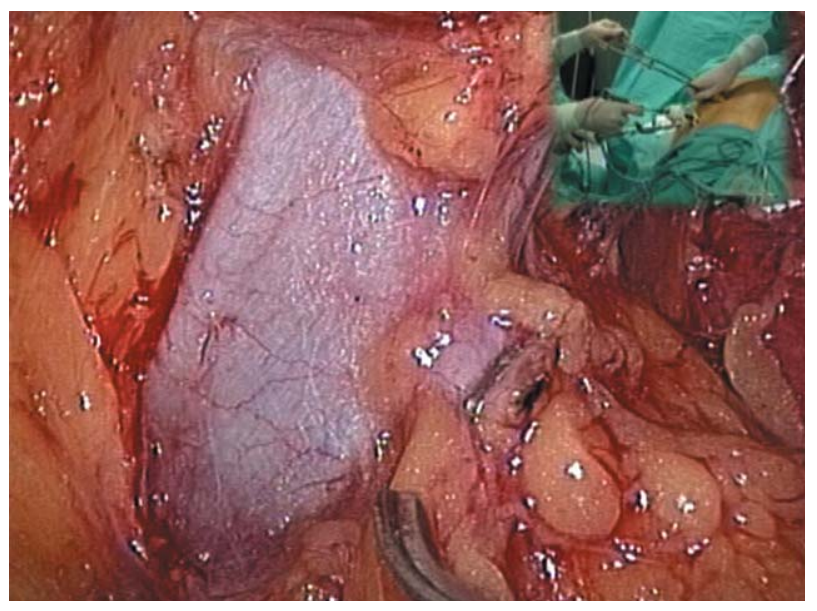

\section{FIGURA 7}

males y seccionamos la vena con endo-GIA o pasamos una ligadura de vena (muy frecuentemente en el lado derecho) con posterior clipaje con hem-o-locks.

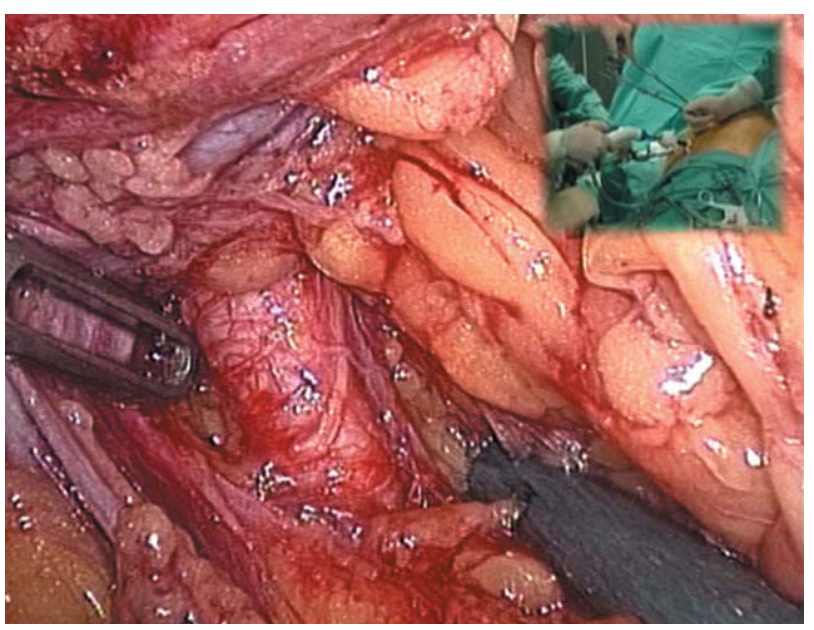

\section{FIGURA 8}

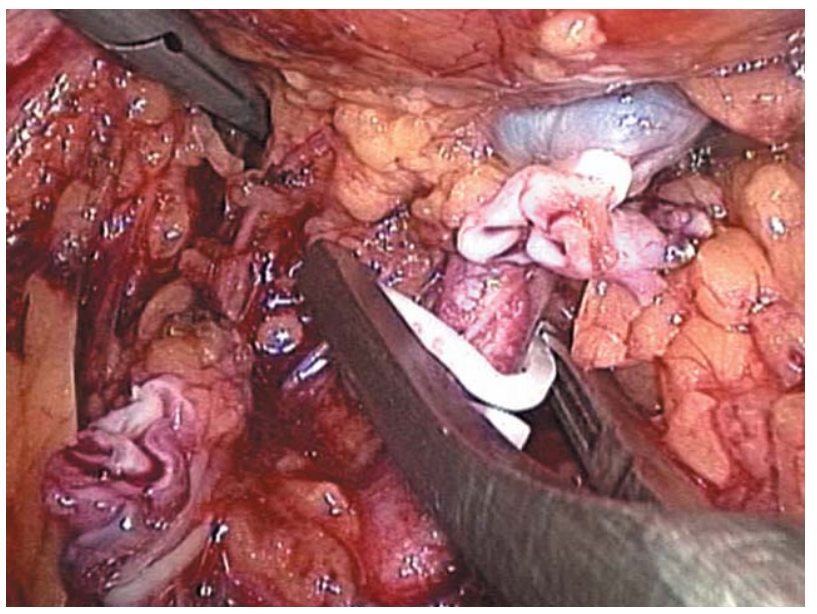

FIGURA 9

h) Sección de uréter; (Fig. 10).

i) Liberación del polo superior; (Fig. 11) siguiendo los criterios oncológicos establecidos, la glándula suprarrenal se incluye o no en la pieza. La maniobra se ve facilitada con la óptica de $30^{\circ}$ y en el lado izquierdo con la movilización previa del eje espleno-pancreático.

j) Exéresis de la pieza embolsada; (Fig. 12).

\section{Via retroperitoneal}

a) Colocación de paciente y cirujanos; lumbotomía clásica en $90^{\circ}$, con las mismas precauciones que en la vía transperitoneal. Los cirujanos se sitúan en la espalda del paciente.

b) Acceso al retroperitoneo; éste se hace con una pequeña incisión de 1-2 cm en la punta de la $12^{\text {a }}$ costilla, con disección digital del espacio retroperitoneal entre el psoas y la cara posterior 


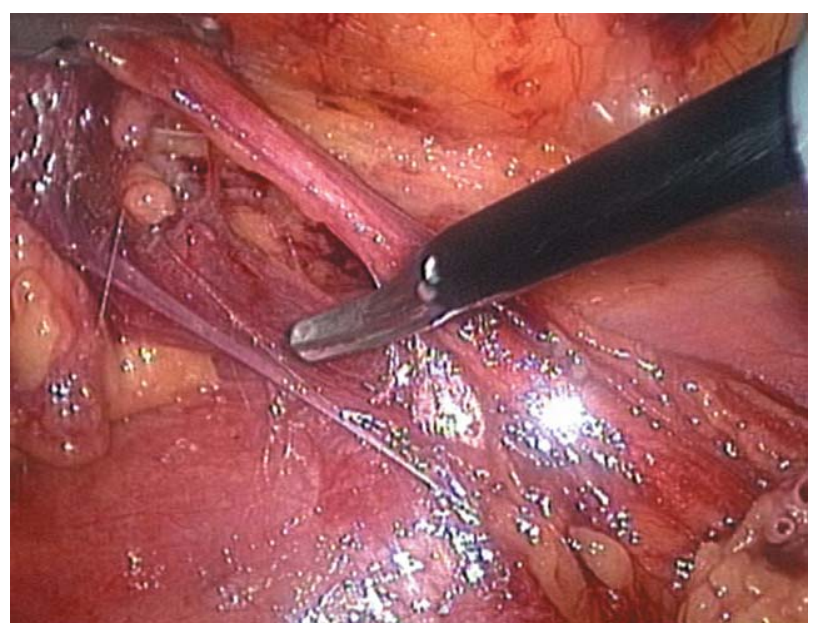

FIGURA 10

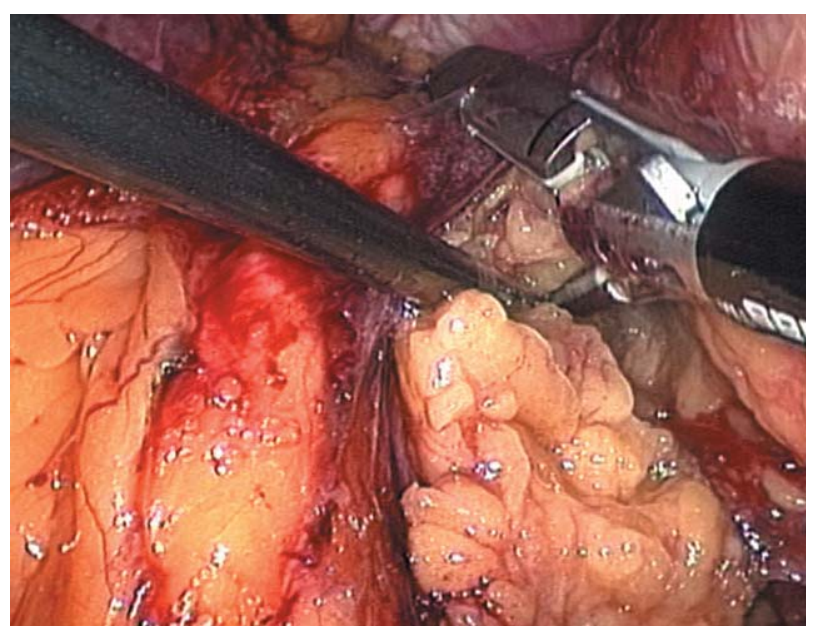

FIGURA 11

de la Gerota. Inserción del balón de dilatación, que se puede hinchar hasta 800 cc de $\mathrm{CO} 2$. Introducción del laparoscopio dentro del balón para comprobar la adecuada creación del espacio. Se retira el balón.

c) Creación del pneumoretroperitoneo e inserción de trócares; se coloca un trócar de $10 \mathrm{~mm}$ a través de la incisión y se insufla el retroperitoneo a $15 \mathrm{~mm} \mathrm{Hg}$. Se colocan 2 trócares más de $10 \mathrm{~mm}$ y 10-12 mm en el borde lateral del erector por debajo de la $12^{\text {a }}$ costilla y entre las líneas axilares media y anterior $3 \mathrm{~cm}$ por encima de la cresta ilíaca.

d) Acceso al hilio; mediante una incisión de la Gerota en contacto con el psoas, se accede, diseca y clipa la arteria renal. La vena renal, anterior a la arteria, es disecada y ligada con endo-GIA o clips.

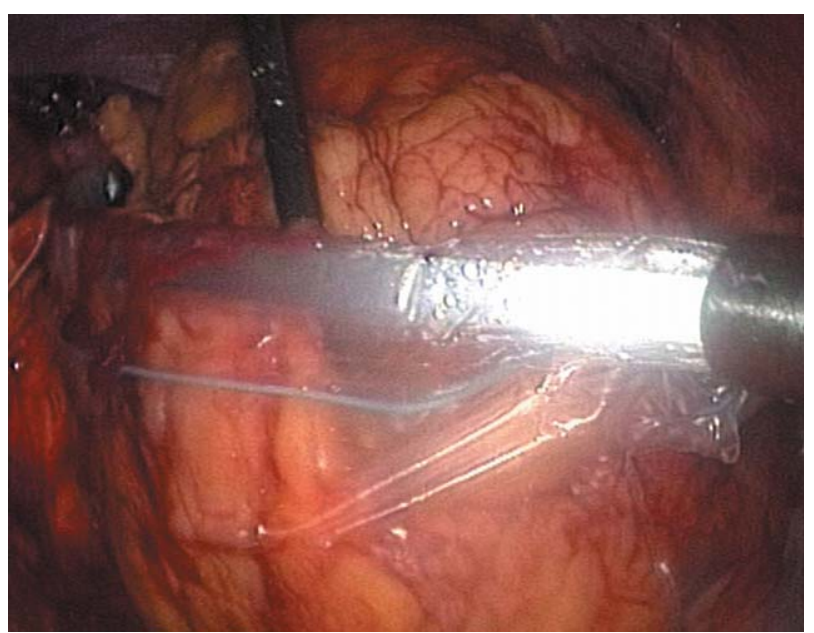

FIGURA 12

e) Disección suprahiliar; siguiendo la musculatura retroperitoneal.

f) Sección de uréter y vasos gonadales.

g) Exéresis de la pieza embolsada.

\section{NRL mano-asistida}

La NRL mano-asistida facilita inicialmente mediante una incisión de 7-9 cm la liberación de adherencias previas si existen. Sus principios quedaron claramente expuestos en los trabajos de Nakada et $a^{11}$. La elección del dispositivo a usar depende de las preferencias del cirujano; en la actualidad hay disponibles 6 dispositivos distintos, entre los que no se han descrito diferencias significativas ${ }^{12}$.

a) Colocación del paciente; no varía respecto a la NRL pura, aunque suele no llegarse a los $90^{\circ}$ de lumbotomía, pudiéndose realizar en decúbito supino con discreta flexión de la mesa quirúrgica.

b) Incisión abdominal y colocación del dispositivo mano-asistida; depende del caso y de las preferencias del cirujano, pero suele escogerse una lejos del retroperitoneo superior para no interferir con los otros dos trócares, siendo la media infraumbilical la más frecuente. A través del dispositivo se colocará la mano no dominante del cirujano.

c) Colocación de trócares; habitualmente se colocan 2 trócares accesorios. El primero de $10 \mathrm{~mm}$ para el laparoscopio y otro de $12 \mathrm{~mm}$ para la mano dominante del cirujano. En el riñón derecho pueden ser necesarios más trócares para rechazar el hígado. 
El resto de pasos quirúrgicos son equivalentes a los descritos para la NRL pura previamente. Esta técnica ha demostrado sus ventajas frente a la cirugía abierta ${ }^{13,14}$. Sus ventajas o inconvenientes frente a la NRL pura han sido también motivo de debate. Sus defensores argumentan el menor tiempo de aprendizaje con la mano asistida $^{15,16}$ así como un menor tiempo quirúrgico ${ }^{17,18}$. Sus detractores han cuestionado su necesidad, la pérdida de espacio por la mano sobretodo en pacientes delgados y el peligro de la habituación a este abordaje para después abordar otro tipo de cirugías en donde no sería necesario su uso. El debate del coste económico de ambas técnicas está sujeto a tantas consideraciones que todo lo publicado en sistemas sanitarios diferentes del nuestro es poco válido.

En nuestra opinión, está técnica puede tener sus indicaciones en determinadas cirugías que se prevean complicadas por el tamaño tumoral, la nefroureterectomía ${ }^{19}$, en la nefrectomía bilateral (decúbito supino) por enfermedad poliquística autosómica dominante ${ }^{20}$ y en la nefrectomía del donante vivo ${ }^{21-23}$.

\section{Extracción de la pieza}

Este último punto es común a ambas vías si no se hace NRL mano asistida y ha sido objeto de debate entre la extracción intacta o el troceado de las piezas. Las supuestas ventajas de ambas posibilidades son la posibilidad de asegurar el estadío patológico de la pieza y las ventajas cosméticas respectivamente. Por el contrario, sus principales argumentos en contra son la necesidad de incisión y la posibilidad de diseminación celular también de forma respectiva.

Recientemente se ha publicado un estudio en el que un avezado urólogo laparoscopista daba a elegir a sus pacientes la posibilidad de trocear (33 pacientes) o sacar su pieza intacta (23 pacientes) argumentándoles las ventajas y los inconvenientes de cada técnica ${ }^{24}$. La mejor cosmética de los casos troceados frente a los abiertos $-1,2 \mathrm{~cm}$ de incisión frente a $7,1 \mathrm{~cm}$ - no se tradujo en una menor analgesia ni estancia. La extracción de piezas intactas fue más rápida, y en el grupo donde no se troceó se estableció en 2 casos un incremento del estadío de cT1/2 a pT3a. Esta cifra se ha valorado retrospectivamente hasta el
$21,9 \%$ de los $\operatorname{casos}^{25}$. Esta eventualidad no supone actualmente ningún cambio en la actitud terapéutica; si en un futuro los protocolos en fase III sobre inmunoterapia autóloga, inhibidores de la angiogénesis $u$ otros tipos de terapia adyuvante a los carcinomas renales de mal pronóstico confirman su utilidad, apurar el diagnóstico patológico de un pT3a sí que será relevante ${ }^{25}$. Los autores del mentado trabajo argumentan que se le debería dar a elegir al paciente qué tipo de extracción prefiere, la posibilidad de realizar incisiones lejanas como un Pfannestield o media infraumbilical poco dolorosas y apuntan los problemas técnicos para triturar piezas, derivados de las características de la única bolsa aprobada para tal fin (LapSac) y de la retirada del mercado del mejor triturador que existía.

\section{COMPLICACIONES}

Las complicaciones en la NRL pueden ser graves y comprenden todas las descritas para la nefrectomía abierta más las inherentes a la cirugía laparoscópica. En general, la tasa de complicaciones mayores se cifra en 5,6\% (5-8,2\%), aunque las cifras pueden variar según cómo los distintos autores clasifican las mismas. Existen grupos que clasifican sus complicaciones en intraoperativas, postoperativas y médicas, ocurriendo respectivamente en $4,7 \%, 6,7 \%$ y $1,8 \%{ }^{26}$. De la misma forma hay descrita una tasa de reconversión a cirugía abierta de 4-7,5\% y una tasa de reintervención ulterior de $1,5 \%{ }^{27}$.

La evolución de las tasas descritas está sujeta en los distintos grupos con mayor experiencia en cirugía laparoscópica a dos fenómenos contrapuestos; en primer lugar, es clara la relación de la experiencia con la disminución de la tasa de complicaciones. Existe un grupo que refiriéndose exclusivamente a la NRL, disminuye su tasa de complicaciones entre la primera y la segunda centena realizadas de $8,3 \% 2,5 \%{ }^{27}$, y esto es extensible al resto de la cirugía laparoscópica $^{28,29}$. En segundo lugar aparece en escena el fenómeno de la estabilización de dichas tasas; a medida que nuevos urólogos se incorporan al programa laparoscópico sufren las complicaciones inherentes a su propia curva de aprendizaje. El balance entre estos dos fenómenos no está analizado específicamente en ningún grupo, aun- 
que es reconocido por todos que tanto la curva de aprendizaje, y de forma paralela las complicaciones, disminuyen cuando aquél se realiza de forma tutorizada y no pionera en un servicio de urología.

Hasta la actualidad se han descrito al menos 4 casos de mortalidad peroperatoria. Un caso de fallo sistémico tras lesión intestinal, un caso de isquemia mesentérica tras NRL no complicada ${ }^{26}$, un caso de lesión de arteria mesentérica y una miocarditis fúngica tras candidemia $\operatorname{tardia~}^{30}$.

\section{Complicaciones relacionadas con la creación del pneumoperitoneo}

Al igual que el resto de la cirugía laparoscópica, la punción con la aguja de Veress en la NRL transperitoneal puede producir lesiones intestinales, vasculares o de órganos sólidos. Igualmente su mala colocación en el espacio preperitoneal puede inducir a errores durante la insuflación produciendo despegamientos peritoneales que induzcan un síndrome vagal severo, habiéndose descrito bradicardias graves. En general se recomienda su punción en fosa iliaca o hipocondrio izquierdo y en fosa iliaca derecha, realizar la comprobación manual y con el flujómetro de su correcto posicionamiento. No se recomienda cuando existen cirugias previas y nunca se usa en la vía retroperitoneal. El desplazamiento al subcutáneo de trócares durante la cirugía puede producir enfisema subcutáneo que raramente tiene importancia clínica durante la NRL.

\section{Complicaciones intestinales}

No se han descrito más tasas de íleo paralítico para la vía transabdominal que para la vía retroperitoneal, pero lógicamente, las temidas lesiones térmicas intestinales no se dan en esta última. Suelen ser producidas por arcos térmicos de instrumentos con defectos en sus vainas o bien por elementos de retracción, muchas veces fuera del campo óptico, y fácilmente pueden pasar inadvertidas. Un abdomen que no recupera la normalidad a los 3-5 días de la NRL debe hacer pensar en esta eventualidad y obligar a una laparoscopia exploradora. De la misma forma se han descrito también complicaciones de la vía biliar.

\section{Complicaciones vasculares}

Son sin lugar a dudas las más frecuentes ${ }^{26}$ y las más temidas. En la NRL siempre hay que tener en cuenta la variabilidad anatómica de los pedículos renales, cifrada hasta en un 30\% en los libros clásicos; sin embargo, y basándose precisamente en la meticulosa visión que ofrece esta vía, no se ha justificado en ningún caso de NRL común ningún tipo de radiología vascular prequirúrgica. Sí que se recomienda examinar con meticulosidad el TAC preoperatorio en un intento de prever dicha eventualidad y de descubrir placas ateromatosas que pudieran comprometer el clipado arterial.

Con experiencia, los desgarros venosos de los vasos dependientes de la vena renal o de ella misma pueden ser controlados laparoscópicamente siguiendo los principios abiertos; compresión, limpieza del campo y sutura o clipado, además del incremento de la presión del pneumoperitoneo a $20 \mathrm{mmHg}$, que siendo temporal, suele ser bien tolerado. Una vez controlado el sangrado, también es recomendable colocar un trócar extra que vuelva a la situación de dos manos al cirujano principal. Sin experiencia, es mejor reconvertir que intentar resolver un sangrado venoso que no se resuelva exclusivamente con compresión.

Se recomienda fehacientemente que el inicio de la curva de aprendizaje de la vía retroperitoneal sea tutorizado, pues las referencias anatómicas pueden no ser tan claras como en la vía transperitoneal y cualquier giro inadvertido de la cámara inducir a confundir la vena renal con la $\operatorname{cava}^{31}$ y la arteria renal con la aorta ${ }^{32}$. Igualmente, el uso de la óptica de $30^{\circ}$ requiere de una concentración máxima del cámara, y en el caso de no disponer de las modernas cámaras en donde ésta está integrada al cable de luz, su uso debe controlarse con las dos manos para asegurar la correcta orientación en todo momento. Se han descrito secciones de arterias distintas a la renal (mesentérica superior, mesentérica inferior o incluso la arteria renal derecha en el caso de una NRL izquierda) precisamente por el mal uso de la cámara.

Los desgarros arteriales son raros, pero no así que salten los clips aplicados por ejemplo a una arteria con placas de ateroma ${ }^{33}$. Aunque los clips 
con mecanismo antideslizante (ej. hem-o-lock) ofrecen más garantías que los de titanio, pueden saltarse también si no se tiene en cuenta dicha posibilidad y/o se colocan muy cerca del ostium en la aorta. El gancho final de los hem-o.locks también ha sido motivo de sangrado en muchos hilios; siempre hay que controlar que su sellado abarca todo el vaso. Actualmente ya se disponen éstos con un tamaño de $15 \mathrm{~mm}$, que muchos grupos colocan transcutáneamente en la vena renal con su correspondiente aplicador, obviando el uso de Endo-GIAs.

En la nefrectomía, donde el despegamiento es amplio, es más que recomendable comprobar a 8 mmHg de presión que no existe sangrado venoso oculto por el pneumoperitoneo al final de la cirugía y revisar la retirada de los trócares laparoscópicamente con cierre endoscópico de las fascias en todos los trócares de 10 o más mm de diámetro para prevenir las hernias.

\section{Desgarros esplénicos/hepáticos}

El trayecto ciego de los trócares situados en los hipocondrios o las adherencias pueden producir fácilmente desgarros parenquimatosos en estos órganos. Su manejo puede ser conservador si se dispone de bisturí de argón o pegamentos biológicos para controlarlos. El Floseal también ha demostrado su utilidad en estos casos.

\section{Complicaciones en relación con la postura}

La NRL se realiza habitualmente en lumbotomía, y, aunque ésta puede no ser tan forzada como en cirugía abierta, hay que tener en cuenta que la cirugia puede ser al principio de la curva de aprendizaje más larga, por lo que el perfecto almohadillado de los puntos óseos así como evitar extensiones forzadas de la pierna ipsilateral puede evitar neuropraxias en el postoperatorio. Esta es la complicación postoperatoria más frecuente $^{26}$.

\section{Pneumotórax}

Aunque raro, se ha publicado esta grave complicación laparoscópica ${ }^{34}$, lo que supone un colapso pulmonar ipsilateral y desplazamiento mediastínico contralateral por la transmisión de la hiperpresión abdominal. Con experiencia, puede controlarse laparoscópicamente con pun- tos sobre el diafragma, pero es un motivo más que justificado para reconvertir si ello no se consigue.

\section{Síndrome del dolor lumbar crónico ${ }^{35}$}

\section{INNOVACIONES TÉCNICAS}

Al ser una técnica relativamente joven, la NRL es una técnica en desarrollo y por tanto las innovaciones técnicas y complicaciones intraquirúrgicas que van apareciendo facilitan o perfeccionan la técnica continuamente. A continuación relataremos alguna de las recientes aportaciones de distintos grupos.

a) La movilización completa del eje pancreático-esplénico con un despegamiento del bazo hasta sus vasos cortos permite una disección mucho más cuidadosa del polo superior del riñón izquierdo y facilita la exéresis de la suprarrenal izquierda cuando ello es necesario

b) Cada vez son más grupos que recomiendan la óptica de $30^{\circ}$ para la NRL; aunque no es estrictamente necesaria, facilita la disección arterial y de los polos superiores

c) El uso de aspiradores romos como elemento de retracción/disección está muy extendido.

d) La retracción del lóbulo hepático derecho con un elemento de agarre cruzado desde el epigastrio anclándolo a la cara interna de la parrilla costal facilita la disección del polo superior y suprarrenales derechas.

e) No se recomienda colocar muchos clips a las venas accesorias de la renal si se planea colocar posteriormente una endo-GIA para la misma, por la posibilidad de mal funcionamiento al englobar un clip.

f) Para pedículos con arterias ateromatosas se ha propuesto el clipado y sección en bloque de arteria y vena con endo-GIA sin haber objetivado fístulas arterio-venosas este grupo a los 26 meses de seguimiento ${ }^{36}$.

g) En arterias arterioescleróticas evitar poner los clips cerca del ostium de la aorta, zona típica de placa de ateroma que puede hacer saltar el mismo.

h) Si se usa una endo-GIA, se puede usar otra sobre aquella si el clipado es insuficiente.

i) En venas renales cortas y dificiles, se ha descrito la posibilidad de ligado previo para reducir su calibre y que entonces sea posible colocar hem-o-locks ${ }^{37}$. 
j) Se han desarrollado distintos dispositivos canulares para la aplicación laparoscópica de pegamentos biológicos, flo-seal, etc, muy útiles al igual que el bisturí de argón para los desgarros parenquimatosos.

k) La última generación de dispositivos manoasistida permiten trabajar con la mano o con instrumentos laparoscópicos a su través sin que se pierda el pneumoperitoneo, lo que permite sumar los beneficios de ambos abordajes ${ }^{38}$.

1) Se ha descrito la nefrectomía radical usando en todos sus pasos el Ligasure, incluido arteria y vena, ganando en rapidez y obteniendo menores pérdidas sanguíneas ${ }^{39}$. Si bien queda descrita esta alternativa, no podemos recomendar esta técnica como "estándar" para la NRL, siguiendo incluso las recomendaciones comerciales del producto.

m) Se ha descrito la posibilidad de acceder directamente a la arteria renal izquierda a través del ángulo de Treitz en la NRL izquierda para su control antes de la manipulación arterial ${ }^{40}$.

n) Con la aparición de la robótica, también se ha descrito la posibilidad de la NRL asistida por el Da Vinci ${ }^{41}$; sin embargo, en una técnica donde normalmente no hace falta suturar ni ligaduras, es difícil pensar que la robótica añada algo a la laparoscopia convencional, sin tener además en cuenta el importante incremento en costes que esta técnica conlleva.

\section{RESULTADOS ONCOLÓGICOS}

Como se dijo previamente, hemos tenido que esperar el juicio del tiempo para poder aseverar la eficacia de la NRL en cuanto a control sus resul- tados oncológicos. En series con un seguimiento medio de 73 meses, no se han demostrado diferencias entre la NRL y la nefrectomía abierta en tasas de supervivencia libre de enfermedad ni en mortalidad cáncer específica a 5 y 10 años $^{42}$ para los estadíos pT1 y pT2. En el carcinoma renal, tampoco parece existir el riesgo de implantación tumoral en los trócares o en la cavidad; 3 de los 4 casos descritos se han debido a violaciones de los principios oncológicos ${ }^{24}$, y estudios prospectivos realizados en otros tumores como cáncer de colon, tampoco han demostrado relación estadísticamente significativa entre el troceado de la pieza y la diseminación ${ }^{43}$, siempre que aquél se realice técnicamente de forma correcta.

En este apartado podemos hacer referencia al papel de la NRL en el cáncer renal metastático. Inicialmente se apuntó que las ventajas inherentes a la cirugía laparoscópica se traducían en un inicio de la inmunoterapia un mes antes que con la vía abierta, además de hipotetizar una respuesta más favorable del sistema inmune ante la menor agresión quirúrgica, pero el mismo grupo no objetivó posteriormente diferencias en supervivencia cáncer específica ${ }^{44}$. Existen trabajos recientes en los que se confirman ese dato ${ }^{45,46} \mathrm{y}$ en los que no se demuestran diferencias en la respuesta inmune según la nefrectomía se haga abierta o laparoscópica ${ }^{47}$. En este grupo de pacientes cobra aún más importancia la meticulosa selección del caso; es difícil comparar la NRL a la vía abierta, pues ésta siempre se debe reservar para aquellos casos con adenopatías voluminosas, grandes tumores o con importante circulación colateral (Tabla 1).

Tabla 1

Resultados oncológicos

\begin{tabular}{|c|c|c|c|c|c|c|c|}
\hline $\begin{array}{l}\text { Autor } \\
\text { año \% }\end{array}$ & $\stackrel{n}{\text { abierta / LAP }}$ & $\begin{array}{c}\text { SLE } \\
\%\end{array}$ & $\begin{array}{c}\text { SCE } \\
\%\end{array}$ & $\begin{array}{l}\text { SG } \\
\%\end{array}$ & $\begin{array}{l}\text { SCE } \\
\text { pT1 }\end{array}$ & $\begin{array}{l}\text { SCE } \\
\text { pT2 }\end{array}$ & $\begin{array}{c}\text { Segto. } \\
(\mathrm{m})\end{array}$ \\
\hline Chan`01 & $54 / 67$ & - & $75 / 86$ & - & - & - & $44 / 36$ \\
\hline Portis` 02 & $69 / 64$ & $91 / 92$ & 92/98 & $88 / 81$ & 95/97 & $87 / \mathbf{1 0 0}$ & $70 / 54$ \\
\hline Saika`03 & $68 / 95$ & $87 / 91$ & - & - & 94/94 & - & $65 / 40$ \\
\hline Wille' 04 & 82 & - & 96.3 & - & - & - & 23.5 \\
\hline Rosales` 04 & 100 & 100 & - & - & $100(n=81)$ & $100(n=18)$ & $21+/-10$ \\
\hline
\end{tabular}

SLE; supervivencia libre enfermedad, SCE; supervivencia cáncer específica, SG; supervivencia global. 


\section{NRL EN GRUPOS DE RIESGO}

\section{Ancianos}

Debido al progresivo envejecimiento de la población, los mejores índices de salud y el aumento de incidencia de tumores renales nos vamos a encontrar progresivamente más tumores en pacientes por encima de los 70. ¿Se mantienen todas las ventajas de la NRL en este grupo etario? Este ha sido un tema analizado por varios grupos últimamente. Matin et al. realizan un estudio retrospectivo de cirugía renal/suprarrenal de 192 pacientes menores de 65 años versus 207 pacientes mayores de 65 a.; no encuentran diferencias en complicaciones, objetivando solo un día más de estancia hospitalaria en los mayores ${ }^{48}$. Dos estudios retrospectivos publicados en 2004 sólo objetivan diferencias en el ASA y en la estancia algo más prolongada ${ }^{49,50}$, pero tampoco diferencias en complicaciones.

En general, se entiende que una vez adquirida una buena destreza laparoscópica, este grupo etario se beneficia igual sino más de la NRL versus la cirugía abierta. Incluso ha habido grupos en recomendar de elección esta técnica en pacientes por encima de 80 años ${ }^{51}$.

\section{ASA III/IV}

De forma similar al grupo previo, los pacientes ASA III/IV han sido motivo de estudio para valorar la repercusión de la NRL; en nuestra experiencia corresponden a el $46 \%$ de los pacientes en los que se hemos realizado NRL. En un estudio comparativo entre 26 casos de nefrectomía abierta, 8 de NRL mano asistida y $13 \mathrm{NRL}$ en pacientes ASA III/IV, las tres tardaban lo mismo, manteniendo la NRL menores requerimientos de analgesia y coste ${ }^{52}$. En este grupo de pacientes es donde no solo la experiencia del urólogo es vital en reducir el tiempo quirúrgico al máximo, sino la del anestesista implicado en el equipo laparoscópico, realizando un buen manejo de la oliguria e hipercapnias inducidas por la laparoscopia, evitando sobrehidrataciones inadvertidas y las consecuentes

Tabla 2 hemodilución e insuficiencia cardiaca congestivas, son de importancia vital para la realización de la NRL.

\section{Obesos}

También este grupo de pacientes es cada vez más frecuente no solo en EEUU (aproximadamente $25 \%$ de la población obesa) sino en nuestro propio país. El $31 \%$ de nuestros pacientes a los que se le ha realizado un NRL tienen un indice de masa corporal mayor de $30 \mathrm{~m}^{2} / \mathrm{kg}$. En la Tabla 2 se muestran distintos trabajos publicados al respecto de este grupo. En general se objetiva como tampoco se obtienen diferencias en tasas de complicaciones y estancias, aunque existen resultados dispares en cuanto al tiempo quirúrgico y las pérdidas sanguíneas entre los diferentes grupos ${ }^{48,53-55}$. Particularmente creemos que la creación del pneumoperitoneo puede ser más complicada en estos pacientes, pero muchas veces la gran cavidad abdominal y el hecho que la mayoría del exceso de grasa se acumula normalmente en el subcutáneo y no en el retroperitoneo, hacen que la NRL propiamente dicha no sea más difícil en los pacientes obesos, que se benefician claramente de incisiones pequeñas, incluso en casos de obesidades extremas ${ }^{56}$.

En este grupo de pacientes se han realizado una serie de recomendaciones ${ }^{57}$ que enumeramos a continuación:

a) Colocación de aguja de Verres y trócares de forma perpendicular para evitar trayectos tangenciales que puedan fugar y producir enfisema.

b) Almohadillado cuidadoso de los puntos óseos.

Nefrectomía radical laparoscópica en obesos (IMC>30 m²/ $\mathrm{kg}$ )

\begin{tabular}{|c|c|c|c|c|}
\hline $\begin{array}{l}\text { Autor año } \\
(\mathrm{NR}>00)\end{array}$ & $\begin{array}{l}\text { Obeso/ } \\
\text { No }\end{array}$ & $\begin{array}{l}\text { Trans/ } \\
\text { Retro }\end{array}$ & $\begin{array}{l}\text { Variables asociadas } \\
\text { a la obesidad }\end{array}$ & $\begin{array}{l}\text { Variables no asociadas } \\
\text { a la obesidad }\end{array}$ \\
\hline Doublet $^{\top} 00$ & $8 / 47$ & $\mathrm{~T}$ & - & TQ, PS, estancia, compl. \\
\hline Matin 03 & ¿/389 & $\mathrm{T} / \mathrm{R}$ & ASA & Complicaciones \\
\hline Fugita`04 & $32 / 69$ & $\mathrm{~T}$ & ASA & $\begin{array}{l}\text { TQ, PS, estancia, tasa conversión, } \\
\text { analgesia, compl. mayores }\end{array}$ \\
\hline Anast ${ }^{-} 04$ & $40 / 77$ & $\mathrm{~T}$ & Mayor transf. & $\begin{array}{c}\text { Tasa conversión, ASA, analgesia, } \\
\text { TQ, PS y transf. }\end{array}$ \\
\hline
\end{tabular}

TQ; tiempo quiúrgico. PS; pérdidas sanguíneas, T; transperitoneal, R; retroperitoneal 


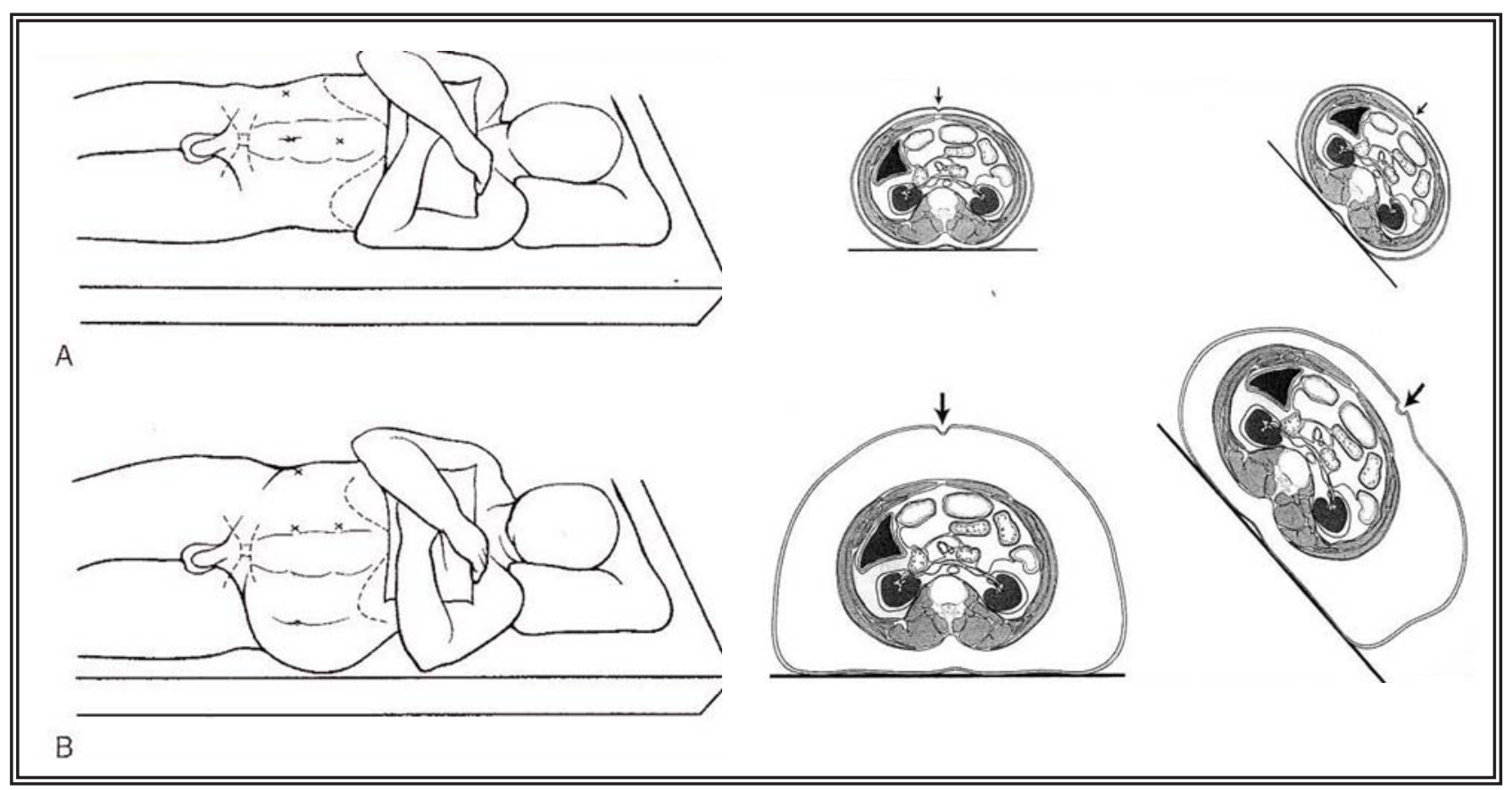

FIGURA 13. Migración lateral de los trócares recomendada en obesos. Tomado de Saika et al Urology 2003; $62(6): 1018$.

c) Migración lateral de la colocación de los trócares para evitar que el desplazamiento del ombligo con el abdomen nos despiste las referencias y coloquemos aquellos lejos del órgano diana (Fig. 13).

d) No usar trócares de tornillo.

e) Existe la posibilidad de usar trócares más largos.

f) Las grandes cavidades abdominales permiten ocasionalmente incrementar la presión de $\mathrm{mmHg}$ sin repercusión sobre el paciente.

g) Cierre laparoscópico de las fascias.

\section{CONCLUSIONES}

Dado que no es científicamente correcto concluir nada en un trabajo de revisión, esperamos simplemente que revisiones como ésta animen a la comunidad urológica a iniciarse en las técnicas laparoscópicas, basándose en los resultados expuestos en éste y otros trabajos. Creemos sinceramente que más allá de la técnica, los requerimientos básicos para iniciarse en la nefrectomía radical laparoscópica pasan por aspectos obvios como tener una gran experiencia en nefrectomias abiertas y realizar una curva de aprendizaje correcta, al ser posible tutorizada, y por aspectos más personales como voluntad de grupo para mejorar la atención a nuestros pacientes.

\section{REFERENCIAS}

1. Clayman RV, Kavoussi LR, Soper NJ, Dierks SM, Meretyk S, Darcy MD, et al. Laparoscopic nephrectomy: initial case report. J Urol. 1991 Aug; 146(2):278-282.

2. Gaur DD, Agarwal DK, Purohit KC. Retroperitoneal laparoscopic nephrectomy: initial case report. J Urol. 1993; 149 (1):103-105.

3. Nakada SY, Moon TD, Gist M, Mahvi D. Use of the pneumo sleeve as an adjunct in laparoscopic nephrectomy. Urology. 1997;49(4):612-613.

4. Rojas D, Rosales A, Palou J, Pascual X, Salvador J, Villavicencio $H$. Bilateral laparoscopic nephrectomy for renal cancer. Arch Esp Urol. 2004 Oct;57(8):844-847.

5. Alcaraz A, Rosales A, Palou J, Caffaratti J, Montlleo M, Segarra J, et al. Living donor nephrectomy for kidney transplantation. Experience in the first two years. Arch Esp Urol. 2004 Dec;57(10):1091-1098.

6. López Cubillana P, Prieto González A, Gómez Gómez G, Cao Avellaneda E, López López AI, Maluff Torres A, et al. Hand-assisted laparoscopic radical nephrectomy vs. open nephrectomy in the treatment of clinically localized renal cell carcinoma. Comparative study. Arch Esp Urol. 2004 Oct:57(8):833-837.

7. Fariña Pérez LA, Delgado C, Dos Santos J. Oxidized cellulose (Surgicel) mimics an abscess after laparoscopic partial nephrectomy" Actas Urol Esp. 2004;28(1):54-56.

8. Nambirajan T, Jeschke S, Al-Zahrani H, Vrabec G, Leeb K, Janetschek G. Prospective, randomized controlled study: transperitoneal laparoscopic versus retroperitoneoscopic radical nephrectomy.Urology. 2004 Nov;64(5):919-924.

9. Desai MM, Strzempkowski B, Matin SF, Steinberg AP, Ng C, Meraney AM, et al. Prospective randomized comparison of transperitoneal versus retroperitoneal laparoscopic radical nephrectomy.J Urol. 2005 Jan;173(1):38-41.

10. Steinberg AP, Finelli A, Desai MM, Abreu SC, Ramani AP, Spaliviero M, et al. Laparoscopic radical nephrectomy for large (greater than $7 \mathrm{~cm}$, T2) renal tumors.J Urol. 2004 Dec;172(6 Pt 1):2172-2176. 
11. Nakada SY. Hand-assisted laparoscopic nephrectomy. J Endourol 1999;13(1):9-14; discussion 14-15.

12. Stifelman MD, Nieder AM. Prospective comparison of hand-assisted laparoscopic devices.Urology. 2002 May;59 (5):668-672.

13. Nakada SY, Fadden P, Jarrard DF, Moon TD. Hand-assisted laparoscopic radical nephrectomy: comparison to open radical nephrectomy.Urology. 2001 Oct;58(4):517-520.

14. Lee SE, Ku JH, Kwak C, Kim HH, Paick SH. Hand assisted laparoscopic radical nephrectomy: comparison with open radical nephrectomy.J Urol. 2003 Sep;170(3):756-759.

15. Marguet CG, Young MD, L'Esperance JO, Tan YH, Ekeruo WO, Preminger GM, et al. Hand assisted laparoscopic training for postgraduate urologists: the role of mentoring. $\mathrm{J}$ Urol. 2004 Jul; 172(1):286-9.

16. Gaston KE, Moore DT, Pruthi RS. Hand-assisted laparoscopic nephrectomy: prospective evaluation of the learning curve. J Urol 2004;171(1):63-67.

17. Wolf JS, Jr, Moon TD, Nakada SY. Hand assisted laparoscopic nephrectomy: comparison to standard laparoscopic nephrectomy. J Urol 1998;160(1):22-27.

18. Kercher KW, Joels CS, Matthews BD, Lincourt AE, Smith TI, Heniford BT. Hand-assisted surgery improves outcomes for laparoscopic nephrectomy.Am Surg. 2003 Dec;69(12):1061-1066.

19. Chueh SC, Chen J, Hsu WT, Hsieh MH, Lai MK. Hand assisted laparoscopic bilateral nephroureterectomy in 1 session without repositioning patients is facilitated by alternating inflation cuffs. J Urol. 2002 Jan; 167(1):44-47.

20. Jenkins MA, Crane JJ, Munch LC. Bilateral hand-assisted laparoscopic nephrectomy for autosomal dominant polycystic kidney disease using a single midline HandPort incision. Urology. 2002 Jan;59(1):32-36.

21. Wolf JS Jr, Marcovich R, Merion RM, Konnak JW. Prospective, case matched comparison of hand assisted laparoscopic and open surgical live donor nephrectomy. J Urol. 2000 Jun;163(6): 1650-1653.

22. Kercher K, Dahl D, Harland R, Blute R, Gallagher K, Litwin D. Hand-assisted laparoscopic donor nephrectomy minimizes warm ischemia. Urology. 2001 Aug;58(2):152-156.

23. Velidedeoglu E, Williams N, Brayman KL, Desai NM Campos L, Palanjian M, et al. Comparison of open, laparoscopic, and hand-assisted approaches to live-donor nephrectomy. Transplantation. 2002 Jul 27;74(2):169172.

24. Hernández F, Rha KH, Pinto PA, Kim FJ, Klicos N, Chan TY, et al. Laparoscopic nephrectomy: assessment of morcellation versus intact specimen extraction on postoperative status.J Urol. 2003 Aug; 170(2 Pt 1):412-5.

25. Cohen DD, Matin SF, Steinberg JR, Zagone R, Wood CG. Evaluation of the intact specimen after laparoscopic radical nephrectomy for clinically localized renal cell carcinoma identifies a subset of patients at increased risk for recurrence. J Urol. 2005 May; 173(5):1487-1490; discussion 1490-1491.

26. Parsons JK, Varkarakis I, Rha KH, Jarrett TW, Pinto PA, Kavoussi LR. Complications of abdominal urologic laparoscopy: longitudinal five-year analysis. Urology. 2004 Jan;63(1):27-32.

27. Simon SD, Castle EP, Ferrigni RG, Lamm DL, Swanson SK, Novicki DE, et al. Complications of laparoscopic nephrectomy: the Mayo clinic experience. Complications of laparoscopic nephrectomy: the Mayo clinic experience. J Urol. 2004 Apr;171(4):1447-1450.
28. Soulie M, Seguin P, Richeux L, Mouly P, Vazzoler N, Pontonnier F, et al. Urological complications of laparoscopic surgery: experience with 350 procedures at a single center.J Urol. 2001 Jun;165(6 Pt 1):1960-1963.

29. Vallancien G, Cathelineau X, Baumert H, Doublet JD, Guillonneau B. Complications of transperitoneal laparoscopic surgery in urology: review of 1,311 procedures at a single center.J Urol. 2002 Jul;168(1):23-26.

30. Steinberg AP, Finelli A, Desai MM, Abreu SC, Ramani AP, Spaliviero M, et al. Laparoscopic radical nephrectomy for large (greater than $7 \mathrm{~cm}$, T2) renal tumors.J Urol. 2004 Dec; $172(6$ Pt 1):2172-2176.

31. McAllister M, Bhayani SB, Ong A, Jaffe W, Malkowicz SB, VanArsdalen $\mathrm{K}$, et al. Vena caval transection during retroperitoneoscopic nephrectomy: report of the complication and review of the literature. J Urol. 2004 Jul;172(1):183-5.

32. Sautter T, Haueisen H, Stierli P, Kwiatkowski M, Recker F. A severe complication of retroperitoneoscopic nephrectomy.J Urol. $2001 \mathrm{Feb}$;65(2):515-516.

33. Baek M, Chun H, Oh SJ, Kim HH. Open conversion from laparoscopic nephrectomy: slippage of surgical clips ligating the renal artery affected by atherosclerosis. J Urol. 2004 Jan; 171(1):333-334.

34. Waterman BJ, Robinson BC, Snow BW, Cartwright PC, Hamilton BD, Grasso M. Pneumothorax in pediatric patients after urological laparoscopic surgery: experience with 4 patients. J Urol. 2004 Mar;171(3):1256-1258; discussion 1258-1259.

35. Oefelein MG, Bayazit Y. Chronic pain syndrome after laparoscopic radical nephrectomy. J Urol. 2003 Nov; 170(5): 1939-1940.

36. Rapp DE, Orvieto MA, Gerber GS, Johnston WK 3rd, Wolf JS Jr, Shalhav AL. En bloc stapling of renal hilum during laparoscopic nephrectomy and nephroureterectomy. Urology. 2004 Oct;64(4):655-659. Review.

37. Janetschek G, Bagheri F, Abdelmaksoud A, Biyani CS, Leeb K, Jeschke S. Ligation of the renal vein during laparoscopic nephrectomy: an effective and reliable method to replace vascular staplers. J Urol. 2003 Oct;170(4 Pt 1):1295-1297.

38. Ponsky LE, Cherullo EE, Banks KL, Greenstein M, Streem $\mathrm{SB}$, Klein EA. Laparoscopic radical nephrectomy: incorporating advantages of hand assisted and standard laparoscopy.J Urol. 2003 Jun;169(6):2053-2056.

39. Leonardo C, Guaglianone S, De Carli P, Pompeo V, Forastiere E, Gallucci M. Laparoscopic nephrectomy using Ligasure system: preliminary experience. J Endourol. 2005 Oct; 19(8):976-8.

40. Porpiglia F, Terrone C, Cracco C, Renard J, Musso F, Grande S, et al. Direct access to the renal artery at the level of treitz ligament during left radical laparoscopic transperitoneal nephrectomy. Eur Urol. 2005 Aug;48(2): 291-295. Epub 2005 Apr 11.

41. Klingler DW, Hemstreet GP, Balaji KC. Feasibility of robotic radical nephrectomy-initial results of single-institution pilot study. Urology. 2005 Jun;65(6):1086-1089.

42. Permpongkosol S, Chan DY, Link RE, Sroka M, Allaf M, Varkarakis I, et al. Long-term survival analysis after laparoscopic radical nephrectomy. J Urol. 2005 Oct;174(4 Pt 1):1222-1225.

43. Pearlstone DB, Feig BW, Mansfield PF. Port site recurrences after laparoscopy for malignant disease. Semin Surg Oncol. 1999 Jun; 16(4):307-312. 
44. Rabets JC, Kaouk J, Fergany A, Finelli A, Gill IS, Novick AC. Laparoscopic versus open cytoreductive nephrectomy for metastatic renal cell carcinoma. Urology. 2004 Nov;64(5):930-934.

45. Mosharafa A, Koch M, Shalhav A, Gardner T, Logan T, Bihrle R, et al. Nephrectomy for metastatic renal cell carcinoma: Indiana University experience. Urology. 2003 Oct;62(4):636-640.

46. Finelli A, Kaouk JH, Fergany AF, Abreu SC, Novick AC, Gill IS. Laparoscopic cytoreductive nephrectomy for metastatic renal cell carcinoma.BJU Int. 2004 Aug;94(3):291-294.

47. Landman J, Olweny E, Sundaram CP, Chen C, Rehman J, Lee DI, et al. Prospective comparison of the immunological and stress response following laparoscopic and open surgery for localized renal cell carcinoma. J Urol. 2004 Apr;171(4):1456-1460.

48. Matin SF, Abreu S, Ramani A, Steinberg AP, Desai M, Strzempkowski B, et al. Evaluation of age and comorbidity as risk factors after laparoscopic urological surgery.J Urol. 2003 Oct; 170 (4 Pt 1):1115-1120.

49. Varkarakis I, Neururer R, Harabayashi T, Bartsch G, Peschel R. Laparoscopic radical nephrectomy in the elderly.BJU Int. 2004 Sep;94(4):517-520.

50. Cobb WS, Heniford BT, Matthews BD, Carbonell AM Kercher KW. Advanced age is not a prohibitive factor in laparoscopic nephrectomy for renal pathology.Am Surg. 2004 Jun;70(6):537-542.

51. Hsu TH, Gill IS, Fazeli-Matin S, Soble JJ, Sung GT, Schweizer D, et al. Radical nephrectomy and nephroureterectomy in the octogenarian and nonagenarian: comparison of laparoscopic and open approaches.Urology. 1999 Jun;53(6): 1121-1125.
52. Baldwin DD, Dunbar JA, Parekh DJ, Wells N, Shuford MD, Cookson MS. Single-center comparison of purely laparoscopic, hand-assisted laparoscopic, and open radical nephrectomy in patients at high anesthetic risk. J Endourol. 2003 Apr; 17(3): 161-167.

53. Doublet J, Belair G. Retroperitoneal laparoscopic nephrectomy is safe and effective in obese patients: a comparative study of 55 procedures. Urology. 2000 Jul;56(1):63-66.

54. Fugita OE, Chan DY, Roberts WW, Kavoussi LR, Jarrett TW. Laparoscopic radical nephrectomy in obese patients: outcomes and technical considerations.Urology. 2004 Feb;63(2):247-52; discussion 252.

55. Anast JW, Stoller ML, Meng MV, Master VA, Mitchell JA, Bassett WW, et al. Differences in complications and outcomes for obese patients undergoing laparoscopic radical, partial or simple nephrectomy.J Urol. 2004 Dec;172(6 Pt 1):2287-2291.

56. Abreu SC, Kaouk JH, Steinberg AP, Gill IS. Retroperitoneoscopic radical nephrectomy in a super-obese patient (body mass index $77 \mathrm{~kg} / \mathrm{m} 2$ ).Urology. 2004 Jan; 63(1): 175-176.

57. Saika T, Ono Y, Hattori R, Gotoh M, Kamihira O, Yoshikawa $\mathrm{Y}$, et al. Long-term outcome of laparoscopic radical nephrectomy for pathologic T1 renal cell carcinoma.Urology. 2003 Dec;62(6):1018-1023.

Dr. J. Rubio Briones

Servicio de Urología.

Instituto Valenciano de Oncología

Prof. Beltrán Báguena 8

46009. Valencia

E-mail: jrubio@fivo.org 